\title{
A New Approach to the Inverse Frobenius-Perron Problem for Prime Gaps: Logistic-Based Chaotic Maps
}

\author{
ROBERTO N. PADUA \\ ORCID No. 0000-0002-2054-0835 \\ rnpadua@yahoo.com \\ Liceo de Cagayan University \\ Cagayan de Oro city, Philippines \\ MERLIZA F. LIBAO \\ ORCID No. 0000-0002-7380-7951 \\ mer_liza@yahoo.com \\ University of Science and Technology \\ of Southern Philippines \\ Cagayan de Oro City, Philippines
}

\begin{abstract}
Given the invariant density or ergodic distribution, we can find some dynamical map $f($. $)$ such that $x_{n+1}=f\left(\mathrm{x}_{\mathrm{n}}\right)$ for which the collection $\left\{\mathrm{x}_{\mathrm{n}}\right\}$ of prime gaps follow the stationary distribution referred to as the Inverse Frobenius-Perron map. We provide an alternative theory for finding such a formula based on chaotic dynamics.
\end{abstract}

Keywords: Prime gaps, invariant density, chaotic map, inverse Frobenius-perron

\section{INTRODUCTION}

A dynamical system $\left\{X_{t}\right\}$ generated by a map $\psi($.$) :$

$$
X_{t+1}=\psi\left(X_{t}\right), \mathrm{t}=0,1,2, \ldots \mathrm{N}
$$

is chaotic if the values $\left\{X_{\mathrm{t}}\right\}$ behave like a random sequence. Devaney (2000) provides a more rigorous definition of chaos which essentially boils down to: (a.) sensitivity to initial conditions, (b.) topological transitivity, and (c.) countably infinite periodic points of all periods. By treating (1) as a pseudo-random sequence for large $N$, one obtains a probability distribution $F($.$) , assumed$ 
absolutely continuous with respect to a Lebesgue measure, that describes the random behavior of the sequence. The invariant distribution $F($.$) is the fixed$ point of a Frobenius-Perron operator L(.) (Pingel, 1989):

$$
\rho_{n+1}(x)=L_{\psi}\left(\rho_{n}(x)\right)
$$

where $\rho_{n}(x)$ is the density at the $n$th iterate. In the case of the logistic map:

$$
X_{t+1}=\psi\left(X_{t}\right)=4 X_{t}\left(1-X_{t}\right), \quad t=0,1,2, \ldots N
$$

The Frobenius-Perron invariant distribution is analytically derived as:

$$
\begin{aligned}
& F(x)=\frac{2 \arcsin (\sqrt{x}}{\pi}, \quad 0<x<1 \\
& f(x)=\frac{1}{\pi \sqrt{x(1-x)}}
\end{aligned}
$$

In many practical situations, the invariant distribution $F($.$) is known and the problem is$ to find the chaotic map $\psi($.$) that generated the values. This is the inverse Frobenius-Perron$ problem (Diakonos, 1997; Nijun-Wei , 2013). Nijun-Wei (2013), Pingel (1989) and others have enumerated four different approaches to the problem: (a.) method of conjugation, (b.) differential equation approach, (c.) Pingel's approach and (d.) matrix approach. In this Chapter, we introduce an approach that is based on the dynamics of a known chaotic map $\psi($.$) and a$ known invariant distribution $\mathrm{F}($.) to construct an unknown chaotic map $\theta($.$) whose invariant$ distribution $H($.$) is known.$

The approach uses the inverse transform theorem which states that if $F(x)$ is the distribution of a random variable $X$, then:

$$
U=F(x) \underset{\sim}{d} U(0,1)
$$

is uniformly distributed on $(0,1)$. Hence, if $H(y)$ is the distribution of another random variable $\mathrm{Y}$, then

$$
U=F(x)=H(y) \underset{\sim}{d} U(0,1)
$$

Equation (5.5) then allows us to connect the dynamics of $Y$ with the dynamics of $X$. That is,

$$
H\left(Y_{t}\right)=F\left(X_{t}\right)=U_{t} \underset{\sim}{d} U(0,1) \text { for each } t
$$

and so,

$$
Y_{t}=H^{-1}\left(F\left(X_{t}\right)\right)
$$




\section{Chaotic Maps Derived by Conjugation of an Auxillary Map}

Let $I=\{x: x \in[0,1]\}$ and $\psi: I \rightarrow I$ be given by Equation (2). Let $J=\{y: y \in(0, \infty)\}$ and $\theta: J \rightarrow J$ be an unknown chaotic map:

$$
Y_{t+1}=\theta\left(Y_{t}\right)
$$

The invariant distribution of (2) is given by Equation (3) while the invariant distribution of $\left\{Y_{t}\right\}$ is a known distribution $H(y)$.

Theorem 1: Let $\psi: I \rightarrow I$ be a chaotic map with invariant distribution $F($.$) and let$ $\theta: J \rightarrow J$ be another chaotic map with invariant distribution $H($.$) . Then:$

$$
\theta=\left(F^{-1} * H\right)^{-1} * \psi *\left(F^{-1} * H\right)
$$

Note that if $\mathbf{G}=\boldsymbol{F}^{-\mathbf{1}} * \boldsymbol{H}$, then: $\boldsymbol{\theta}=\boldsymbol{G}^{-\mathbf{1}} * \boldsymbol{\psi} * \boldsymbol{G}$.

Proof. From (6) and (7):

$$
\begin{aligned}
Y_{t}= & H^{-1}\left(F\left(X_{t}\right)\right)=H^{-1}\left(F\left(\psi\left(X_{t-1}\right)\right)=H^{-1}\left(F \left(\psi\left(F^{-1}\left(U_{t-1}\right)\right)\right.\right.\right. \\
& =\left(F \left(\psi \left(F^{-1}\left(H\left(Y_{t-1}\right)\right)\right.\right.\right.
\end{aligned}
$$

By Theorem 1, $\theta($.$) is obtained by conjugation of \psi($.$) . The map \theta($.$) inherits the$ dynamical characteristics of $\psi($.$) . This is not the same as the conjugation approach of Nijun-Wei$ (2015) who derived $\theta($.$) independently of \psi($.$) .$

Let $H($.$) be the exponential distribution:$

$$
H(y)=1-\exp (-\lambda y), \lambda>0, y>0
$$

where $\lambda \approx \frac{1}{\log (N)}$ for large $N$. Equation (8) arises as an approximation to the distribution of prime gaps:

$$
Y_{n}=P_{n+1}-P_{n}, n=1,2,3, \ldots N
$$

where $P_{n+1}$ and $P_{n}$ are consecutive primes (Cramer, 1936; Selberg, 1948; Yamasaki and Yamasaki, 1991). On the dynamics of prime gaps (Libao 2016 dissertation), we established that the gaps $\left\{Y_{n}\right\}$ form a chaotic sequence with a periodic point of period 3. Li and Yorke (1975) demonstrated that if a system has a period 3 point, then the system is chaotic. 
From (5) and (8), we have:

$$
\frac{2 \arcsin \left(\sqrt{X_{t}}\right.}{\pi}=1-\exp \left(-\lambda Y_{t}\right)
$$

Hence,

$$
Y_{t}=\frac{-1}{\lambda} \ln \left(1-\frac{2}{\pi} \arcsin \sqrt{\psi\left(X_{t-1}\right)}\right)
$$

However,

$$
\begin{gathered}
X_{t-1}=\sin ^{2}\left(\frac{\pi}{2}\left(1-\exp \left(-\lambda Y_{t-1}\right)\right) .\right. \\
\text { Let } \varphi_{t-1}=\frac{\pi}{2}\left(1-\exp \left(-\lambda Y_{t-1}\right)\right) \text {, then: } \\
Y_{t}=\frac{-1}{\lambda} \ln \left(1-\frac{2}{\pi} \arcsin \sqrt{4 X_{t-1}\left(1-X_{t-1}\right)}\right) \\
Y_{t}=\frac{-1}{\lambda} \ln \left(1-\frac{4}{\pi} \varphi_{t-1}\right)
\end{gathered}
$$

Equation (9) further simplifies to:

Hence,

$$
Y_{t}=\frac{-1}{\lambda} \ln \left|-1+2 \exp \left(-\lambda Y_{t-1}\right)\right|
$$

$$
\theta(y)=\frac{-1}{\lambda} \ln |-1+2 \exp (-\lambda y)|
$$

Theorem 2. Let $\psi(x)=4 x(1-x)$ with invariant distribution:

$$
f(x)=\frac{1}{\pi \sqrt{x(1-x)}}, 0<x<1
$$

and let $\theta(y)$ be the dynamical map for the prime gaps with invariant distribution:

then,

$$
h(y)=\lambda \exp (-\lambda y), \quad \lambda \approx \frac{1}{\log (N)},
$$

$$
\theta(y)=\frac{-1}{\lambda} \ln |-1+2 \exp (-\lambda y)|
$$

The dynamical map $\theta$ mimics the random pattern generated by the logistic map. In fact:

Corollary 1. The fixed points $X_{0}=0$ and $X_{0^{\prime}}=\frac{3}{4}$ of the logistic map are mapped onto the fixed points $Y_{0}=0$ and $Y_{0^{\prime}}=\frac{\ln (3)}{\lambda}$ of the map $\theta$. 
Proof. We show that the points $Y_{0}=0$ and $Y_{0^{\prime}}=\frac{\ln (3)}{\lambda}$ are fixed points of $\theta$. For $Y_{0}=0$, we have:

$$
\theta(0)=\frac{-1}{\lambda} \ln \left(-1+2 \exp (-\lambda(0))=\frac{-1}{\lambda} \ln (-1+2(1))=\frac{-1}{\lambda}(0)=0 .\right.
$$

For the second point, we note that $-1+2 \exp (-\lambda y)<0$, , hence:

$$
\frac{-1}{\lambda} \ln [-(-1+2 \exp (-\lambda y))]=y
$$

It follows that:

$$
\begin{gathered}
1-2 \exp (-\lambda y)=\exp (-\lambda y) \\
\exp (-\lambda y)=\frac{1}{3} \\
y=\frac{\ln (3)}{\lambda} \\
\text { and } \theta\left(\frac{\ln (3)}{\lambda}\right)=\frac{\ln (3)}{\lambda}
\end{gathered}
$$

Figure 1 shows the plot of $\theta(y)$ against $y$ for $N=100,000,000$.

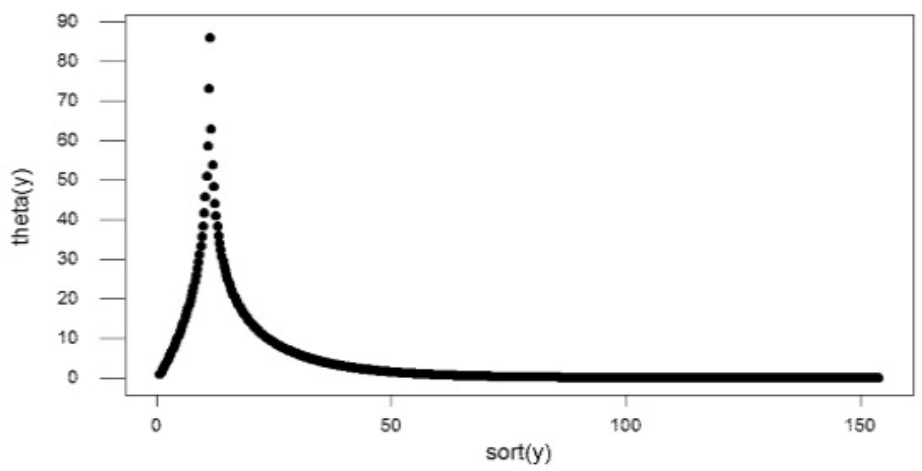

Figure 1. Theta $(y)$ versus $y$.

Figure 2 shows the phase diagram of $Y(t+1)$ versus $Y(t)$ using the initial condition $\mathrm{Y}_{0}=.06$. 


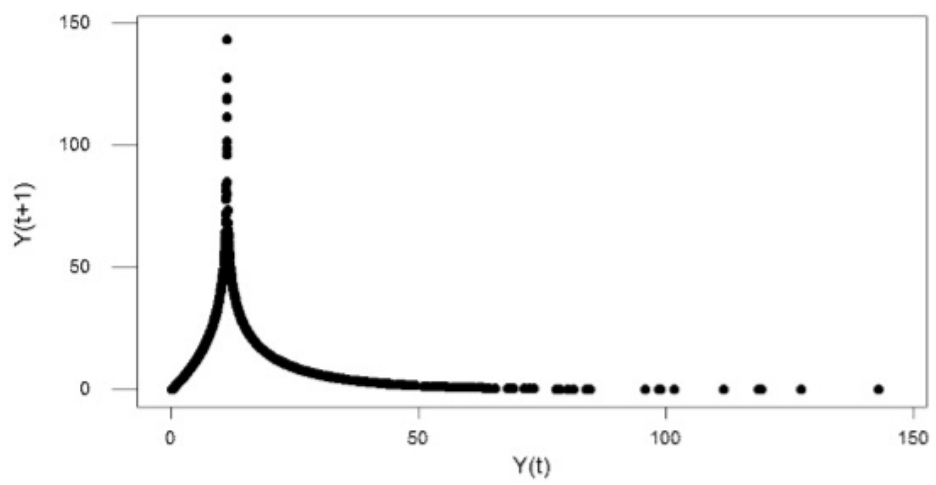

Figure 2. Phase diagram of $Y(t+1)$ versus $Y(t)$.

On the other hand, we also viewed the phase diagram for $E\left(Y_{\mathrm{t}+1} \mid \mathrm{Y}_{\mathrm{t}}=\mathrm{y}\right)$ versus $Y_{\mathrm{t}}=\mathrm{y}$ for the actual prime gaps up to gap $=18$ as shown in Figure 3:

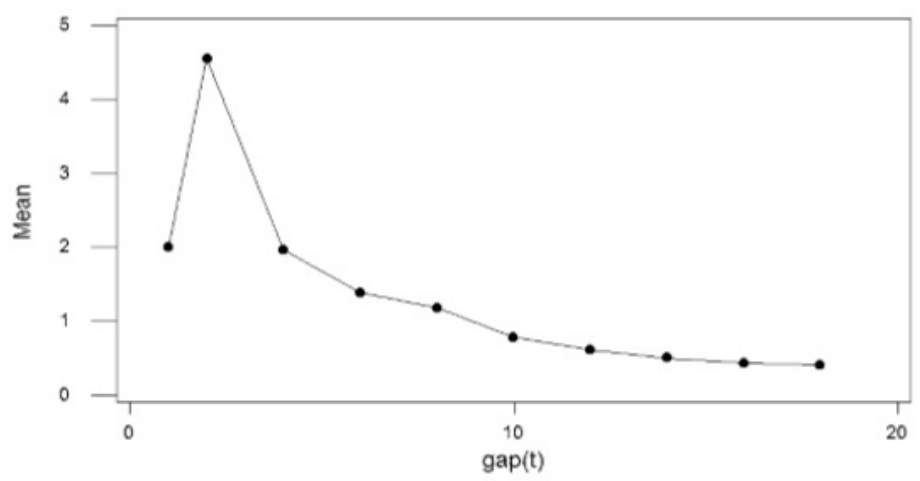

Figure 3. Phase diagram of $E(Y t+1 \mid Y t)$ and $Y t$.

We verify that the pseudo-random numbers generated by $\theta\left(Y_{t-1}\right)$ follow an exponential distribution. This is contained in Theorem 3.

Theorem 3. Let $X_{t+1}=4 X_{t}\left(1-X_{t}\right)$ with ergodic distribution Beta $\left(\frac{1}{2}, \frac{1}{2}\right)$. Then:

$$
Y_{t}=-\frac{1}{\lambda} \ln \left|1-\frac{2 \arcsin \left(\sqrt{x_{t}}\right)}{\pi}\right|
$$

has an exponential distribution with rate parameter $\lambda$. 
Proof: For each $t$,

$$
F\left(x_{t}\right)=\frac{2\left[\arcsin \left(\sqrt{x_{t}}\right)\right]}{\pi}=u_{t}=1-e^{-\lambda y_{t}} \text { where } u_{t} \underset{\sim}{d} U(0,1) .
$$

The result follows.

Theorem 3 suggests the following algorithm:

\section{Algorithm:}

1. Choose an initial value $X_{0} \in[0,1]$ away from a fixed point of the logistic map;

2. Compute $X_{t+1}=4 X_{t}\left(1-X_{t}\right), t=0,1,2, \ldots, n$;

3. Compute $Y_{t}=-\frac{1}{\lambda} \ln \left|1-\frac{2 \arcsin \left(\sqrt{x_{t}}\right)}{\pi}\right|, \quad t=0,1,2, \ldots, n$;

4. Return $Y_{t}$

The specific prime gaps sequence $\{2,2,4,2,4,2,4,6, \ldots\}$ is just one of the infinitely many possible paths that $\left\{Y_{t}\right\}$ can take. Note, however, that $X_{t}=\psi\left(X_{t-1}\right)$ and given step 3 of the algorithm, the behavior of $\left\{Y_{t}\right\}$ is completely determined by the behavior of $\psi($.$) . Likewise, given$ the dynamical character of $\left\{X_{t}\right\}$, the initial value $X_{0}$ completely specifies the trajectory of the system.

\section{Theoretical Bound for the Mean Absolute Error}

Consider:

$$
\frac{1}{n} \sum_{t=0}^{n}\left|\left(Y_{t}-Y_{t^{\prime}}\right)\right|
$$

representing the average divergence of the two trajectories of the chaotic paths $\left\{Y_{t}\right\}$ and $\left\{Y_{t}\right\}$. The Lyapunov characteristic exponent of a dynamical system is a quantity that characterizes the rate of separation of infinitesimally close trajectories (Bryant et al., 1990). Two trajectories diverge at a rate given by:

$$
d(y) \approx \exp (\delta(y))\left|d\left(y_{0}\right)\right|=k \exp (\delta(y))
$$

where $d\left(y_{0}\right)$ is the initial separation of the trajectories. The quantity $\delta(y)$ is the Lyapunov exponent. This is given by:

$$
\delta(y)=\lim _{n \rightarrow \infty} \frac{1}{n} \sum_{n=0}^{\infty} \ln \left(\left|\theta^{\prime}(y)\right|\right)
$$

Since the invariant distribution of $\left\{Y_{t}\right\}$ is known, we can compute:

$$
\delta(y)=\int_{0}^{\infty} \ln \left|\theta^{\prime}(y)\right| d H(y)
$$


It is easy to see that:

$$
\theta^{\prime}(y)=\frac{-2}{\exp (\lambda y)-2}
$$

And

$$
\left|\theta^{\prime}(y)\right|=\frac{2}{\exp (\lambda y)-2} \text { for large } y
$$

Hence,

$$
\delta(y)=\int_{0}^{\infty} \ln \left|\theta^{\prime}(y)\right| d H(y)=\ln (2)-\int_{1}^{\infty} \frac{\ln |u-2|}{u^{2}} d u, u=\exp (\lambda y)
$$

The integral on the right converges to

$$
\begin{aligned}
& \delta(y)=\int_{0}^{\infty} \ln \left|\theta^{\prime}(y)\right| d H(y)=\ln (2)-\int_{1}^{\infty} \frac{\ln |u-2|}{u^{2}} d u \\
& \approx \ln (2)-\left[\int_{3}^{\infty} \frac{-1}{2 u} d u+\int_{3}^{\infty} \frac{1}{2(u-2)} d u\right] \\
& \approx \ln (2)+\frac{1}{2} \int_{3}^{\infty} \frac{1}{u} d u-\frac{1}{2} \int_{3}^{\infty} \frac{d u}{(u-2)} \\
& \approx \ln (2)+\frac{1}{2} \ln \left(\frac{u}{u-2}\right)_{3}^{\infty}=\ln (2)-\frac{1}{2} \ln (3)
\end{aligned}
$$

when the singularity at $u=2$ is avoided. In this case, the Lyapunov exponent is roughly $\delta=0.143841$. Consequently,

$$
\frac{1}{n} \sum_{t=0}^{n}\left|\left(Y_{t}-Y_{t^{\prime}}\right)\right| \sim \mathrm{k} \exp (\delta) \log (n)=1.15470 k \log (n) \text { where }|k| \leq 1
$$

The Lyapunov exponent for the chaotic logistic map is $\ln (2)$ (Pesin, 1977). We observe that the Lyapunov exponent obtained for the chaotic map $\theta(y)$ is less than $\ln (2)$ indicating that $\theta(y)$ is less chaotic than $\psi(x)$.

The smallest prime gap is $y=2$ and for this $u=\exp (\lambda y)=1.11469$. The Cauchy principal value of the full integral is:

$$
-\int_{1}^{\infty} \frac{\ln |u-2|}{u^{2}} d u=\frac{1}{2} \ln \left(\frac{u}{|u-2|}\right)_{1}^{\infty}=0
$$

and:

$$
\delta(y)=\int_{0}^{\infty} \ln \left|\theta^{\prime}(y)\right| d H(y)=\ln (2)-\int_{1}^{\infty} \frac{\ln |u-2|}{u^{2}} d u=\ln (2), \quad u=\exp (\lambda y)
$$

from which we recover the Lyapunov exponent of the chaotic logistic map. It follows that:

$$
\frac{1}{n} \sum_{t=0}^{n}\left|\left(Y_{t}-Y_{t^{\prime}}\right)\right| \sim k \exp (\delta) \log (n)=2 k \log (n),|k| \leq 1
$$




\section{Theoretical Absolute Error}

Next, we consider the magnitude of the difference between the $n$th prime gap $(Y n)$ and the nth prime gap prediction $(Y n)$ :

$$
\begin{aligned}
\left\|Y_{n}-Y_{n^{\prime}}\right\| & \leq\left|\log (n)-\ln \left(\frac{2}{\pi} \arcsin (\sqrt{.5000})\right) \ln (n)\right| \\
\left\|Y_{n}-Y_{n^{\prime}}\right\| & \leq|1-0.693147| \ln (n)=0.306853 \ln (n) \\
\text { where } X_{0} & =0.50000=E(X) \text { of the arcsine distribution (4). }
\end{aligned}
$$

Theorem 4. Let $Y_{n}$ be the $n$th prime gap and let $Y_{n^{\prime}}$ be the estimated nth prime gap based on the chaotic map $\theta(y)$ obtained by conjugation of the chaotic logistic map $\psi(x)$. Then:

$$
\left\|Y_{n}-Y_{n^{\prime}}\right\| \leq|1-0.693147| \ln (n)=0.306853 \ln (n)
$$

\section{Numerical Simulation}

Let $\{Y t\}=\{1,2,2,4,2,4,2,4,6,2,6, \ldots\}$ be the natural sequence of prime gaps starting from the first gap of 1 . Using the exponential approximation to the distribution of prime gaps with:

$$
f(y)=\lambda e^{-\lambda y}
$$

and

$$
\lambda \approx \frac{1}{\log (n)}
$$

then,

$$
x_{t}=\sin ^{2}\left(\frac{\pi}{2}\left(1-e^{-\lambda y_{t}}\right)\right), n=10^{8} .
$$

We obtain the initial condition for the chaotic logistic map by substituting $y_{0}=1$ to obtain:

$$
x_{0}=0.00687
$$

where $\log (100,000,000)=18.4207$. The sequence induced by the logistic map with this initial condition is given by:

$$
x_{t+1}=4 x_{t}\left(1-x_{t}\right), \quad x_{0}=0.00687
$$

These will produce the sequence $\left\{y_{t}{ }^{\prime}\right\}=\left\{1, y_{1}{ }^{\prime} y_{2}{ }^{\prime}, \ldots\right\}$ which has an exponential distribution with the same rate parameter $\lambda$ as the original distribution of $y_{t}$.

The mean absolute error computed for this sequence is:

$$
M A E=\frac{1}{1228} \sum_{t=0}^{1227}\left|Y_{t}-Y_{t}^{\prime}\right|=14.127
$$


for the first 1,228 prime gaps.

We then proceeded to examine the behavior of the sequence in the neighborhood of $x_{0}=0.00687$. Table 1 shows the MAE obtained for various choices of initial values in the neighborhood of $x_{0}$.

Table 1. MAE for Various Initial Values Near $\mathrm{X}_{0}=0.00687$

\begin{tabular}{|c|c|}
\hline MAE & Initial Values $\mathbf{X}_{0}$ \\
\hline 14.467 & 0.00287 \\
\hline 14.417 & 0.00387 \\
\hline 14.320 & 0.00487 \\
\hline 14.369 & 0.00587 \\
\hline 14.126 & 0.00687 \\
\hline 14.342 & 0.00700 \\
\hline 14.303 & 0.00800 \\
\hline 14.615 & 0.00900 \\
\hline 14.268 & 0.01000 \\
\hline 14.414 & 0.01500 \\
\hline 14.716 & 0.02000 \\
\hline 14.072 & 0.02500 \\
\hline 14.311 & 0.03000 \\
\hline 14.458 & 0.03300 \\
\hline 14.186 & 0.03400 \\
\hline 14.345 & 0.03500 \\
\hline 14.608 & 0.03362 \\
\hline
\end{tabular}

The minimum value of the MAE occurs at $\mathrm{x}_{0}=0.025$ with $\mathrm{MAE}=14.072$. The graph of initial values versus MAE is shown below:

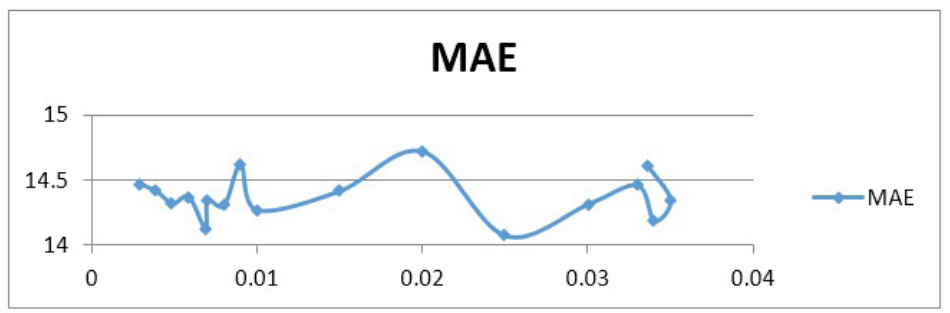

Figure 4. Mean Absolute Errors for Various Initial Values. 
The histogram for the optimal sequence of gaps generated from the initial condition $x=0.025$ is shown below to be exponential as desired:

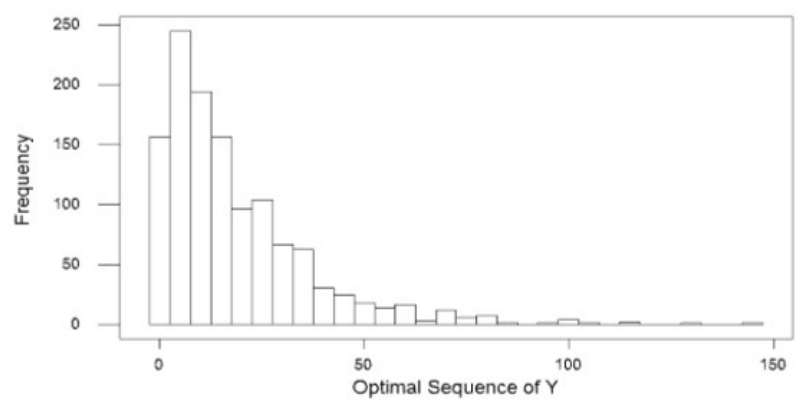

Figure 5. Histogram for the Optimal Sequence of Y Generated from X $=0.025$.

Figure 4 shows that the objective function (MAE) for the minimization problem has several local minima within $(.003, .035)$. However, we also note that the minimum among the local minima satisfies:

$$
\operatorname{Min}_{y_{t}{ }^{\prime}}\left(\min \frac{1}{1228} \sum_{t=0}^{1227}\left|Y_{t}-Y_{t}^{\prime}\right| \leq 0.763923 \log (n) \approx \gamma \log (n)\right.
$$

where $\gamma=$ Ramanujan - Landau constant $=.764223$ or using $(10), k=.661577$.

The initial condition used previously was based on the fixed point $x=0$ and the initial prime gap of $y=1$. We can also explore the other unstable fixed point at $x=.75$ and use the following relation

$$
Y_{t}=-\frac{1}{\lambda} \ln \left|\frac{2 \arcsin \left(\sqrt{x_{t}}\right)}{\pi}\right|
$$

Table 2 shows the results of the exploration around the neighborhood of 0.75 . We find that the objective function has a unique minimum at around $X_{0}=0.739$ and:

$$
\begin{gathered}
\min \frac{1}{1228} \sum_{t=0}^{1227}\left|Y_{t}-Y_{t}^{\prime}\right| \leq 0.7366712 \log (n) \approx \gamma^{1.13655} \log (n) \text { or using }(10), \\
k=0.6370763
\end{gathered}
$$


where $y$ is the Ramanujan-Landau constant earlier given. Table 2 shows the results of the exploration.

Table 2. MAE for Initial Values Near an Unstable Fixed Point $\mathrm{X}_{0}=0.75$

\begin{tabular}{|c|c|}
\hline Initial Values & MAE \\
\hline 0.7300 & 15.941 \\
\hline 0.7350 & 15.292 \\
\hline 0.7370 & 14.476 \\
\hline 0.7390 & 13.570 \\
\hline 0.7400 & 13.729 \\
\hline 0.7401 & 15.323 \\
\hline 0.7420 & 15.532 \\
\hline 0.7600 & 15.960 \\
\hline
\end{tabular}

Figure 6 shows the mean absolute errors for various initial values used.

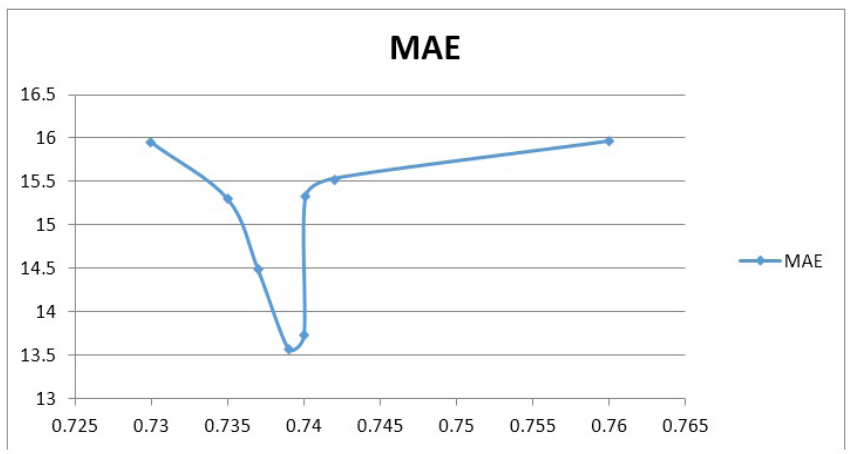

Figure 6. MAE around the neighborhood of $x=0.75$.

Figure 7 shows the histogram of the optimal sequence of gaps generated with an initial condition of $x_{0}=0.739$. 


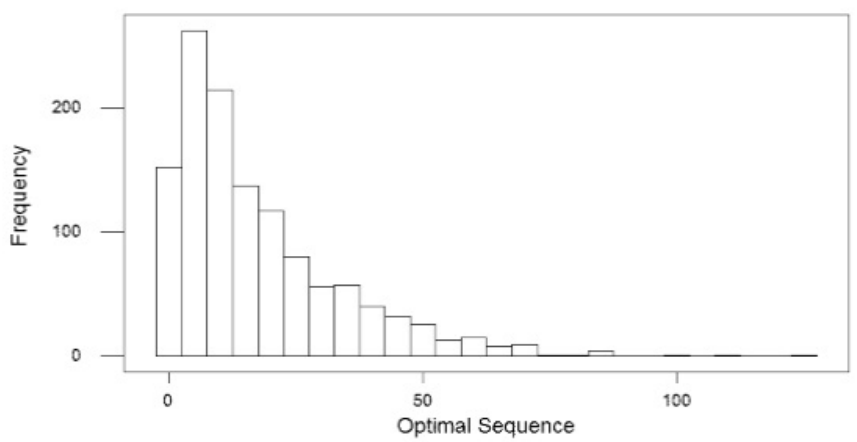

Figure 7. Histogram of the Optimal Sequence of Gaps with Initial Condition $\mathrm{X}_{0}=0.739$

\section{Absolute Errors}

Table 3 shows the estimated nth prime $P_{n}$ and the actual nth prime $\mathrm{P}_{n}$ with their absolute errors and relative absolute errors:

Table 3. Absolute Error for the Estimation of the nth Prime

\begin{tabular}{|l|l|l|l|l|c|l|}
\hline $\mathbf{N}$ & $\mathbf{P}(\mathbf{N}-\mathbf{1})$ & $\begin{array}{l}\text { Actual } \\
\mathbf{P}(\mathbf{N})\end{array}$ & $\begin{array}{l}\text { Estimated } \\
\text { Gap }\end{array}$ & $\begin{array}{l}\mathbf{P}(\mathbf{N})- \\
\text { Estimated }\end{array}$ & ABS(Error) & Relative Abs Error \\
\hline 5000 & 48593 & 48611 & 5.90361 & 48599 & 12 & 0.000246858 \\
\hline 10000 & 104723 & 104729 & 6.38406 & 104729 & 0 & 0.000000000 \\
\hline 15000 & 163819 & 163841 & 6.6651 & 163826 & 15 & $9.15522 \mathrm{E}-05$ \\
\hline 30000 & 350351 & 350377 & 7.14555 & 350358 & 19 & $5.42273 \mathrm{E}-05$ \\
\hline 100000 & 1299689 & 1299709 & 7.98007 & 1299697 & 12 & $9.23284 \mathrm{E}-06$ \\
\hline 200000 & 2750131 & 2750159 & 8.46052 & 2750139 & 20 & $7.27231 \mathrm{E}-06$ \\
\hline 300000 & 4256227 & 4256233 & 8.74156 & 4256236 & 3 & $7.04849 \mathrm{E}-07$ \\
\hline 400000 & 5800057 & 5800079 & 8.94097 & 5800066 & 13 & $2.24135 \mathrm{E}-06$ \\
\hline 500000 & 7368743 & 7368787 & 9.09563 & 7368752 & 35 & $4.74976 \mathrm{E}-06$ \\
\hline 600000 & 8960447 & 8960453 & 9.22201 & 8960456 & 3 & $3.34805 \mathrm{E}-07$ \\
\hline 664578 & 9999971 & 9999973 & 9.29286 & 9999980 & 7 & $7.00002 \mathrm{E}-07$ \\
\hline
\end{tabular}

Table 4 compares the estimated nth prime using the dynamical map $\theta(y)$ and the Prime Number Theorem prediction of the nth prime: 
Table 4. PNT versus Chaotic Map Prediction of the nth Prime

\begin{tabular}{|c|c|c|c|}
\hline $\mathbf{N}$ & $\begin{array}{c}\text { Actual Nth Prime } \\
\text { Number }\end{array}$ & $\begin{array}{c}\text { Predicted } \boldsymbol{P}_{\boldsymbol{N}} \\
\text { Using PNT }\end{array}$ & $\begin{array}{c}\text { Chaotic Map Estimate of } \\
\boldsymbol{P}_{\boldsymbol{N}}\end{array}$ \\
\hline 5000 & 48611 & 42586 & 48599 \\
\hline 10000 & 104729 & 92103 & 104729 \\
\hline 15000 & 163841 & 144237 & 163826 \\
\hline 30000 & 350377 & 309269 & 350358 \\
\hline 100000 & 1299709 & 1151293 & 1299697 \\
\hline 200000 & 2750159 & 2441215 & 4250139 \\
\hline 300000 & 4256233 & 3783461 & 5800066 \\
\hline 400000 & 5800079 & 5159688 & 7368752 \\
\hline 500000 & 7368787 & 6561182 & 8960456 \\
\hline 600000 & 8960453 & 7982811 & 9999980 \\
\hline 664578 & 9999973 & 8909936 & \\
\hline
\end{tabular}

Tabular values show that the predictions made using the chaotic dynamics approach are much closer to the actual primes than the predictions using the Prime Number Theorem.

\section{CONCLUSION}

With the concept of deterministic randomness, we modelled the prime gaps as a chaotic dynamical system. Given the invariant density or ergodic distribution, we can find some dynamical map $f\left(\right.$.) such that: $x_{n+1}=f\left(x_{n}\right)$ for which the collection $\left\{x_{n}\right\}$ of prime gaps follow the stationary distribution referred to as the Inverse Frobenius-Perron map. This map is given by

$$
\theta(y)=\frac{-1}{\lambda} \ln |-1+2 \exp (-\lambda y)| .
$$

\section{LITERATURE CITED}

Bryant, P., Brown, R., Abarbanel, H. (1990). "Lyapunov exponents from observed time series”. Physical Review Letters. 65 (13): 1523.

Eckmann, J.P. \& Ruelle, D. (1985). "Ergodic theory of chaos and strange attractors,” Rev. Mod. Phys. 57:617 
Libao, M. \& Padua, R. (2016). "On the dynamics of prime gaps”. Libao dissertation, Ch. 2.

May, Robert M. (1976). "Simple mathematical models with very complicated dynamics,” Nature, vol.261, 459-467.

Pesin, Y. (1977). "Characteristic Lyapunov exponents and smooth ergodic theory,” Russian Math. Surveys, 32:55

Wei, Nijun (2015). Solutions of the Inverse Frobenius-Perron Problem, http://spectrum. library.concordia.ca/980195 